\title{
discs large regulates somatic cyst cell survival and expansion in Drosophila testis
}

\author{
Fani Papagiannouli ${ }^{1}$, Bernard M Mechler ${ }^{1}$ \\ ${ }^{I}$ Department of Developmental Genetics, DKFZ-ZMBH Allianz, INF 581, Heidelberg 69120, Germany
}

\begin{abstract}
Gonad development requires a coordinated soma-germline interaction that ensures renewal and differentiation of germline and somatic stem cells to ultimately produce mature gametes. The Drosophila tumour suppressor gene discs large (dlg) encodes a septate junction protein functioning during epithelial polarization, asymmetric neuroblast division, and formation of neuromuscular junctions. Here, we report the role of $d l g$ in testis development and its critical function in somatic cyst cells (SCCs). In these cells dlg is primarily required for their survival and expansion, and contributes to spermatocyte cyst differentiation. Cell death primarily occurred in SCCs at the end of spermatogonial amplification at a time when Dlg becomes restricted in wild-type (wt) testes to the distal somatic cells capping the growing spermatocyte cysts. RNAi depletion of $\mathrm{dlg}$ transcripts in early SCCs fully prevented testis development, whereas depletion in late SCCs resulted in a breakdown of spermatocyte cyst structure and germ cell individualization. Specific dlg expression in SCCs resulted in developmental rescue of $d l g$ mutant testes, whereas its expression in germ cells exerted no such effect. $d l g$ overexpression in wt testes led to spermatocyte cyst expansion at the expense of spermatogonial cysts. Our data demonstrate that $\mathrm{dlg}$ is essentially required in SCCs for their survival, expansion, and differentiation, and for the encapsulation of the germline cells.
\end{abstract}

Keywords: discs large, Drosophila, testis formation, somatic cyst cells

Cell Research (2009) 19:1139-1149. doi: 10.1038/cr.2009.71; published online 23 June 2009

\section{Introduction}

In Drosophila melanogaster the development of embryonic gonad results from the coalescence of two specialized cell types, migrating germ cells, and somatic mesodermal cells [1]. Following gonad coalescence during which the germ cells are surrounded by somatic mesodermal cells extending cellular processes between them, the gonad becomes compacted and spherical [2]. The development of male and female gonads already differs at the time of gonad coalescence, as male-specific somatic mesodermal cells join the posterior of the male gonad, while the equivalent cells die by apoptosis in females [2]. A hub is formed at the anterior of the late

\footnotetext{
Correspondence: Bernard M Mechler

Tel: +496221424502; Fax: +496221424552

E-mail: dev.genetics@dkfz-heidelberg.de

Abbreviations: dlg (discs large); GSCs (germline stem cells); L (larval stage); lgl (lethal (2) giant larvae); SCs (spermatogonial cysts); SCCs (somatic cyst cells); scrib (scribble); SSCs (somatic stem cells); wt (wild type) Received 9 February 2009; revised 31 March 2009; accepted 2 April 2009; published online 23 June 2009
}

embryonic male gonad and consists of a cluster of nondividing apical mesodermal cells, which organize the anterior-most germ cells in a rosette arrangement similar to that of germline stem cells (GSCs) in the adult [2]. The first signs of testis organogenesis are detected in first instar larvae (L1) when the germ cells start to expand, whereas those of the ovaries remain quiescent $[2,3]$.

In the niche, the GSCs enclosed in a pair of somatic stem cells (SSCs), are physically attached to the hub cells (Figure 1A) via adherens junctions [4]. Each GSC divides asymmetrically with the mitotic spindle orientated perpendicular to the hub $[5,6]$. One of the daughter cells remains in contact with the hub, inherits the mother centriole, and retains GSC identity, whereas the other daughter cell, called gonialblast, inherits the daughter centriole. This cell is displaced from the hub and initiates differentiation [7-9]. The gonialblast, wrapped by two somatic cyst cells (SCCs) [10], divides mitotically four times to give rise to a cyst of 16 interconnected spermatogonial cells. The SCCs grow without division, become flat, elongate, and form a thin layer around the spermatogonial cyst (SC). The germ cells become transcriptionally highly active and form spermatocytes, 
A
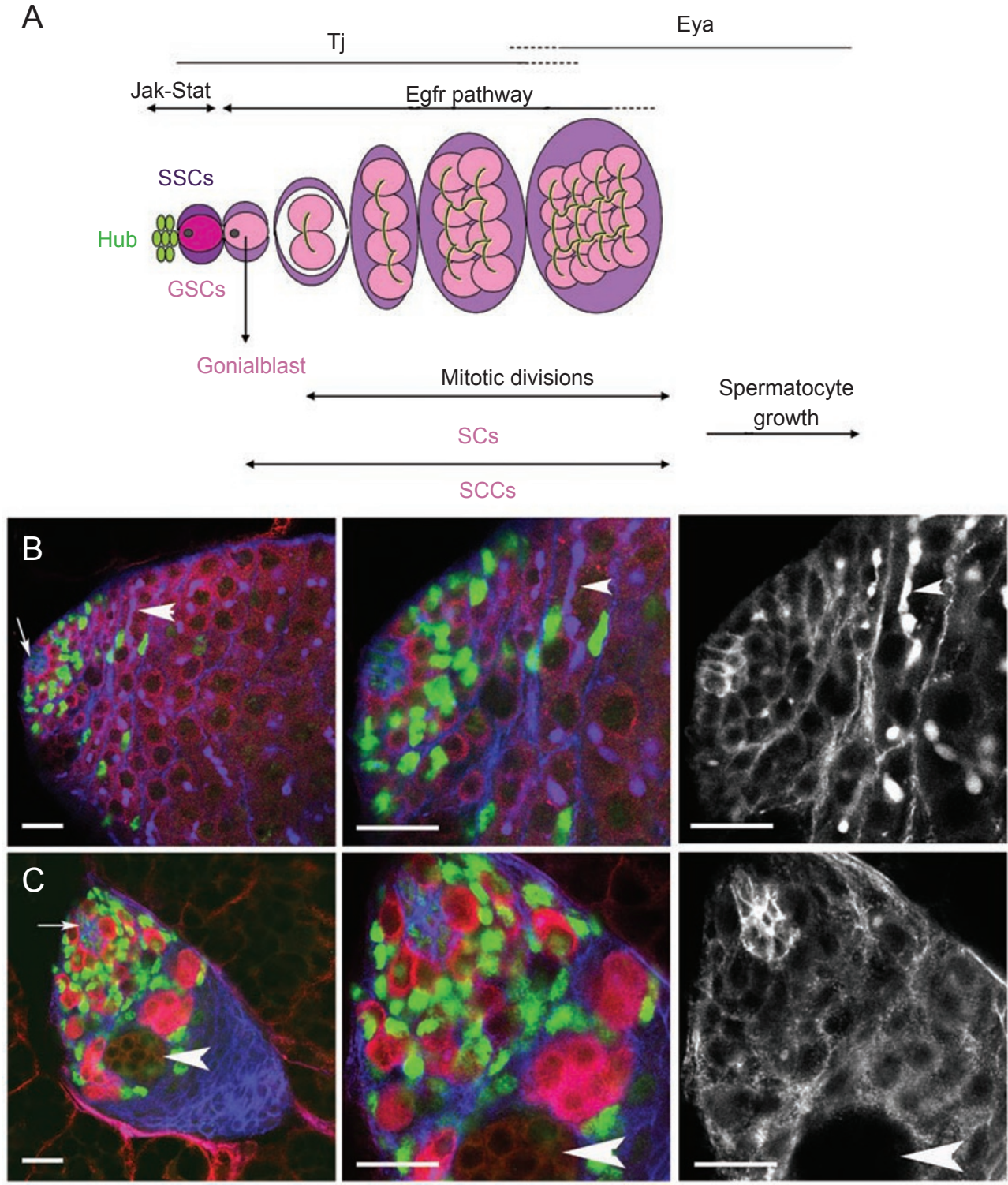

Figure 1 Reduced number of GSCs and SCs in dlg testis. (A) Diagram depicting early spermatogenesis. Eya, Eyes absent; GSCs, germline stem cells; SC, spermatogonial cyst; SCCs, somatic cyst cells; SSCs, somatic stem cells; Tj, traffic jam. The black dots in GSCs and goniablasts and the black-yellow arches in spermatogonial cells represent the spectrosome and the fusome, respectively. Testes from 5-day-old (B) wt and (C) dlg L3 larvae stained for Vasa (red), Tj (green), and Arm+ $\alpha$ Spectrin (blue). Mid panel pictures are enlargements of the hub region shown on left, whereas the right panel pictures show co-staining of Arm and $\alpha$-Spectrin. Testis hub (arrow) is oriented towards the left. Arrowheads in (B) point out the fusomes and arrowheads in C indicate a weak Vasa-stained SC. Bar: $15 \mu \mathrm{m}$.

which over a period of $80 \mathrm{~h}$ will considerably increase in size and ultimately undergo meiosis [11].

The balance between stem cell identity and differentiation results from signals exchanged between the hub, GSCs and SSCs. The hub cells secrete Unpaired (Upd), which activates the Janus kinase (JAK)-signal transducer and activator of transcription (STAT) pathway in adjacent GSCs. In absence of JAK-STAT signaling the GSCs differentiate and are unable to undertake self-renewal.
Conversely, increased expression of JAK-STAT greatly expands the population of GSCs and SSCs $[12,13]$. A further signaling pathway restricting GSC proliferation is mediated by the epidermal growth factor receptor (Egfr), whose inactivation in SSCs leads to an expansion of male GSCs [14]. Similarly, Raf, an Egfr downstream component, is required in SSCs to limit GSC expansion [15]. In testes mutated for the rhomboid homologue stet, the germ cells fail to associate with SSCs [16]. Further- 
more, germ cells recruit SSCs via the ligand Spitz, which binds to Egfr, and acts through the nucleotide exchange factor Vav to regulate the activity of Rac1, a downstream component of the Egfr pathway [17]. In addition, loss of the TGF- $\beta$ signal transducers punt and schnurri in SCCs leads to overproliferation of gonialblasts and spermatogonial cells but not of GSCs [18].

The observation that three well-established tumor suppressor genes, discs large (dlg) [19, 20], scribble (scrib) [21, 22], and lethal (2) giant larvae (lgl) [23], are expressed in the Drosophila embryonic gonads and larval testis [24], prompted us to analyze their function in this tissue. Here, we report that $d l g$ plays a critical role in testis formation during larval development primarily by regulating somatic cell survival.

\section{Results}

\section{Reduced number of GSCs and SCs in dlg larval testes}

As $d l g$ testes were particularly small (both amorphic $d l g^{m 52}$ and hypomorphic $d \lg ^{X 12}$ mutations led to a similar phenotype), we examined which types of cells were affected by $d l g$ inactivation. Similar to wt (wild type) (Figure 1B) the hub of a $d l g$ testis (Figure 1C) appeared normal and consisted of tightly packed cells positively stained for Armadillo (Arm), suggesting that adherens junctions remained intact in the hub region. In contrast, the number of GSCs was reduced in $d l g(4.9 \mathrm{GSCs} /$ testis in 12 testes examined) with respect to wt (8.1 GSCs/testis in eight testes examined). Furthermore, the $d l g$ GSCs were less tightly packed around the hub. Compared to $d l g$, scrib testes showed no sign of degeneration with the exception that they were reduced in size, whereas $l g l$ testes were comparatively normal in size and shape (data not shown). The size of $d l g$ GSCs, gonialblasts, and spermatogonial cells were larger than in wt testes. Graphical determination of the length (L) and width (W) of wt (L $=6.2 \pm 1.0 \mu \mathrm{m}, \mathrm{W}=4.9 \pm 0.4 \mu \mathrm{m})$ and $d l g(\mathrm{~L}=9.5 \pm$ $3.3 \mu \mathrm{m}, \mathrm{W}=6.5 \pm 0.88 \mu \mathrm{m})$ GSCs revealed that the volume of the dlg cells should be two to three times larger than that of wt GSCs. Moreover, dlg germ cells showed a more intense staining of the germ cell-specific marker Vasa, suggesting an accumulation of Vasa proteins in these cells. In addition, we found a marked reduction in the number of 8- or 16-cell cysts in L3 dlg testes. Surviving cysts showed Vasa staining, stronger than in wt. However, few large 16-cell cysts exhibited a much weaker Vasa staining (Figure 1C, arrowhead), suggesting that these cysts were engaged in an apoptotic process. A further characteristic of spermatogonial cells was the gradual disappearance of spectrosomes and fusomes [4, 25], which were absent in L3 $d l g$ testes (right in Figure
$1 \mathrm{~B}$ and C), albeit present in scrib testes (data not shown). These data indicate that multiple defects occurred during early spermatogenesis, leading to SC disappearance and testis atrophy.

As gonad development requires a coordinated somagermline interaction ensuing renewal and differentiation of germline and SSCs, we first studied the distribution of known nuclear markers of somatic cell differentiation, including Traffic-jam (Tj) [26] and Eyes absent (Eya) [27]. In wt testes, $\mathrm{Tj}$ is produced in early SCCs and in elongated SCCs wrapping 2-, 4-, 8-cell cysts (Figure $1 \mathrm{~B})$, whereas Eya is present in SCCs enclosing larger cysts (Figure 2A) [28]. In dlg testes of 3-day-old larvae (Figure 1C), we found that the number of positively stained $\mathrm{Tj}$ cells was comparable to that in wt (Figure $1 \mathrm{~B})$. In contrast, the number of Eya-positive cells was
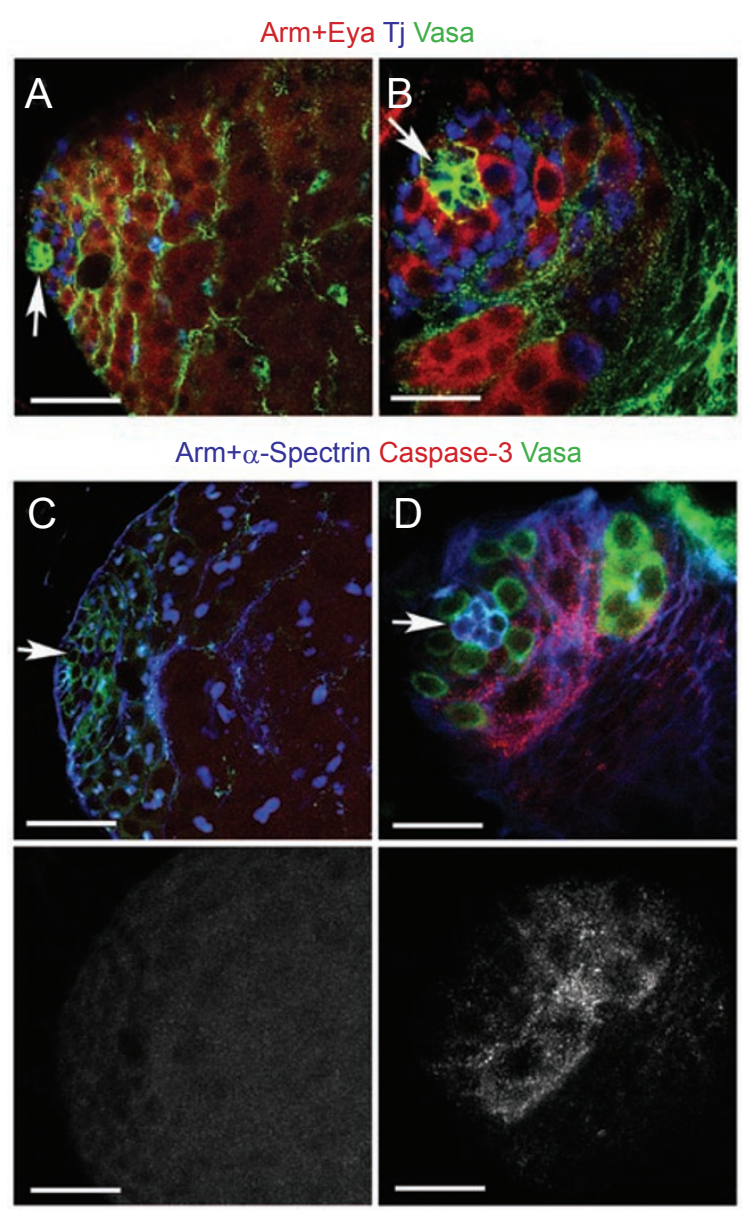

Figure 2 Cell death takes place primarily in late SCCs. (A) wt and (B) dlg testes were stained for Vasa (red), Tj (blue), and Arm+Eya (green). (C) wt and (D) dlg testes stained for activated caspase-3 (red), Vasa (green), and Arm+ $+\alpha$-Spectrin (blue). Lower panel pictures show the caspase- 3 staining. Testis hub (arrow) is oriented towards the left. Bar: $15 \mu \mathrm{m}$. 
strongly reduced in $d l g$ (Figure 2B). These data suggest a developmental arrest occurring when SCs are wrapped by SCCs. Therefore, we determined which type of cells displayed the first sign of cell death by staining $d l g$ testes with rabbit monoclonal anti-active caspase- 3 antibody and found that cell death took place predominantly in SCCs and also affected germ cells in the largest 8- and 16-cell cysts (Figure 2D). Similar results were obtained by using the TUNEL assay (data not shown). Expression of a UAS-p35 transgene encoding a baculovirus homolog of the Drosophila inhibitor of apoptosis protein [29, 30] in the germ line and somatic cells to block caspase activation had no gross effect on the size of $d l g$ testes (data not shown), suggesting a cell death pathway independent of Diap. Although the exact mechanism by which cell death takes place in $d l g$ testes remains to be further determined, our data led us to conclude that $d l g$ function is primarily required in SCCs for their survival and indicate that a somatic $d l g$ function is needed for the expansion and differentiation of germ cells.

The marked degeneration of $d l g$ testis incited us to determine more precisely when the first abnormalities can be detected during testis morphogenesis. As shown in Figure 3, dlg testes already showed signs of degeneration in L1 larvae. These testes were characterized by a smaller number of germ cells, predominantly aggregated around the hub or in its vicinity. In L2 mutant larvae, the defects became more obvious with few GSCs attached to the hub and a small number of larger SCs.

\section{Dlg distribution in differentiating cysts}

To ascertain the role of $d l g$ in SCCs, we investigated the pattern of Dlg distribution during normal testis development. In L1 testis (Figure 4A), Dlg was uniformly associated with the cytoskeletal matrix of all somatic and germ cells, and its distribution overlapped with that of F-actin (data not shown). In L2 (Figure 4B) and L3 testes (Figure 4C-F), we observed a uniform Dlg distribution among the hub cells, in between SSCs and GSCs associated with the hub and at the interphase of SCCs and germ cells in the newly formed 2-, 4-, and 8-cell SCs (Figure 4C-E). However, the pattern dramatically changed within the 16-cell SCs in which Dlg decorated the distal SCC. In this cell the Dlg staining was associated with the plasma membrane forming a relatively smooth line with short indentations at contact sites with neighbouring germ cells (Figure 4F). This pattern was limited to a crescent capping a portion of the somatic cell located near the surface of the testis. In more internal locations, Dlg was essentially present at junctions between cyst cells. As shown in Figure 4G-J co-immunostaining with anti-Dlg and anti-Eya revealed that the Eya-positive nuclei (arrows)

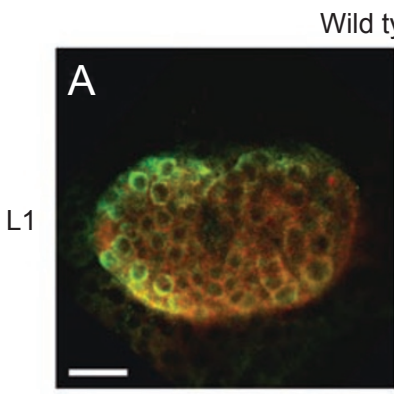

Wild type
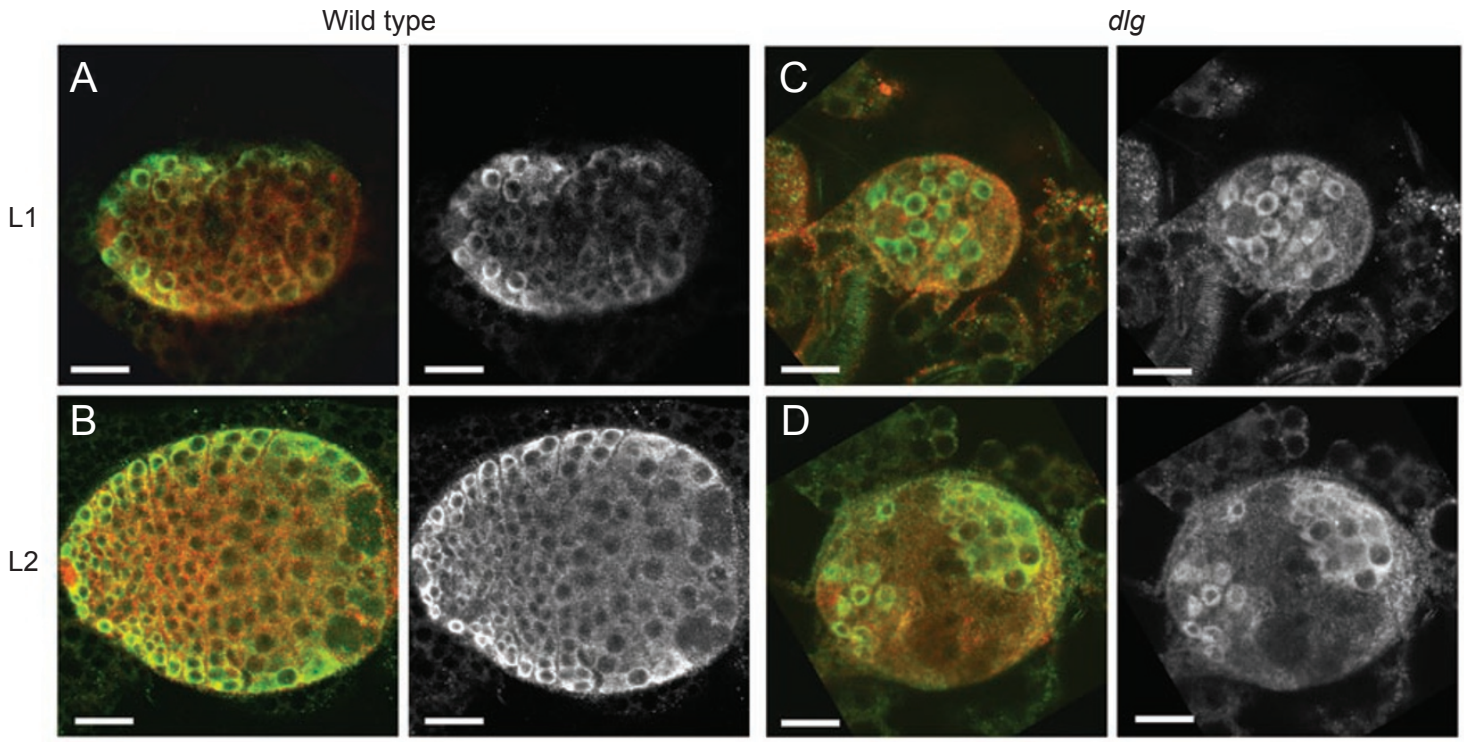

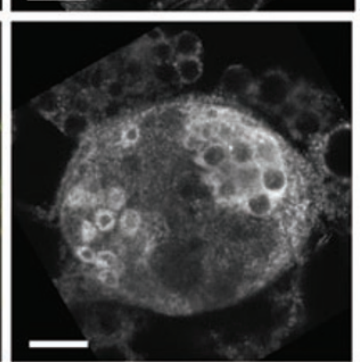

Figure 3 Reduced size of $d l g$ testes and reduced number of germ cells. (A, B) Wt and (C, D) dlg testes from (A, C) L1 and (B, D) L2 larvae. Cell contours were visualized by Scribble I (red) and germ cells were detected by using Vasa antibodies (green). Testis hub is oriented towards the left. The size of dlg testes was smaller than in wt and depleted in germ cells as detected with Vasa antibodies. In dlg testes, germline stem cells (GSCs) were loosely attached to the hub and the overall number of germ cells and SCs were markedly reduced in comparison to wt testes. Pictures on the right of (A-D) display the Vasa staining. Bar: $15 \mu \mathrm{m}$. 

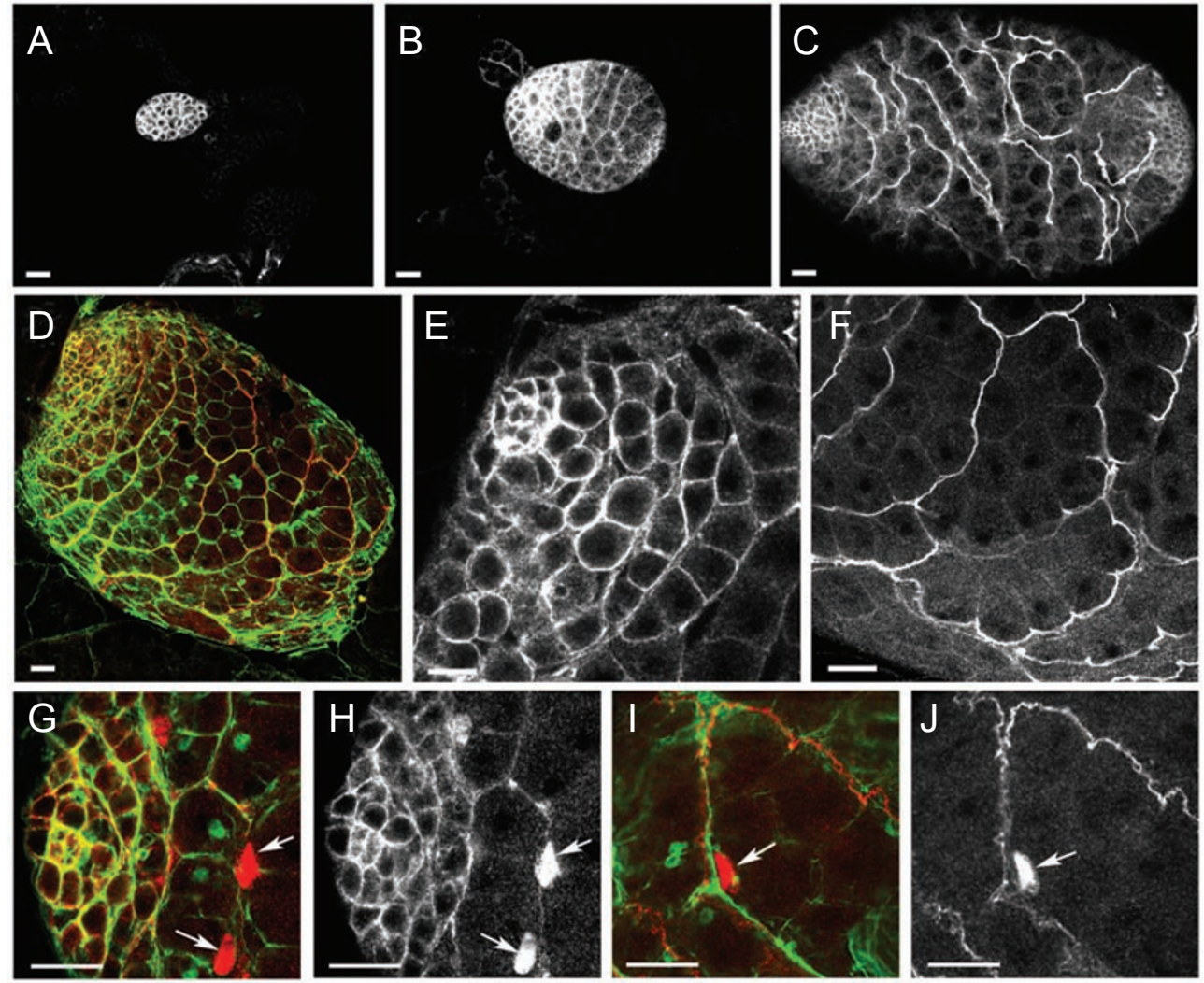

Figure 4 Pattern of Dlg distribution in testis during larval development. Wt testes stained for Dlg in (A) L1 (36 h after egg laying (AEL)), (B) L2 (48-h AEL), and (C) L3 (96-h AEL) larvae. Testis hub is oriented towards the left. (D-F) 5-day-old wt testis stained for F-actin with phalloidin (green) and immunostained for Dlg (red). (E) and (F) are enlargements of (D), displaying Dlg staining in the hub region and growing SCs under the surface of the testis, respectively. (G-J) wt testis stained for F-actin with phalloidin (green) and immunostained for Dlg and Eya (red) to visualize better the position of the SCCs. (G and $\mathbf{H})$ Hub region, (I and J) growing spermatocyte cyst. $(\mathbf{H})$ and $(\mathbf{J})$ display only the Dlg and Eya staining of $(\mathbf{G})$ and $(\mathbf{I})$, respectively. Arrows indicate the Eya positively stained nuclei of SCCs. Bar: $15 \mu \mathrm{m}$.

were closely linked to the periphery of germline cysts in association with the Dlg-stained cortical matrix of SCCs. The spatially restricted distribution at the distal edge of growing SCs suggests that Dlg may regulate cyst growth and differentiation.

dlg expression in somatic cells can restore development of dlg mutant testes

To determine more precisely the critical period of $d l g$ function in the interaction between soma and germline, we tested first whether the JAK-STAT-signaling path-

Figure 5 Unpaired drives stem cell proliferation in dlg. (A) wt, (B) UAS-upd/+; nanos-Gal4, (C) dlg, and (D) dlg; UAS-upd/+; nanos-Gal4 L3 testes stained for $\mathrm{Tj}$ (red) and Arm+a-Spectrin (green). Ectopic upd expression in wt and dlg testes led to overproliferation of spectrosome containing cells, indicating stem cell or gonialblast identity, and cells expressing the Tj marker, revealing a somatic identity. Arrows indicate the hub. Bar: $15 \mu \mathrm{m}$.
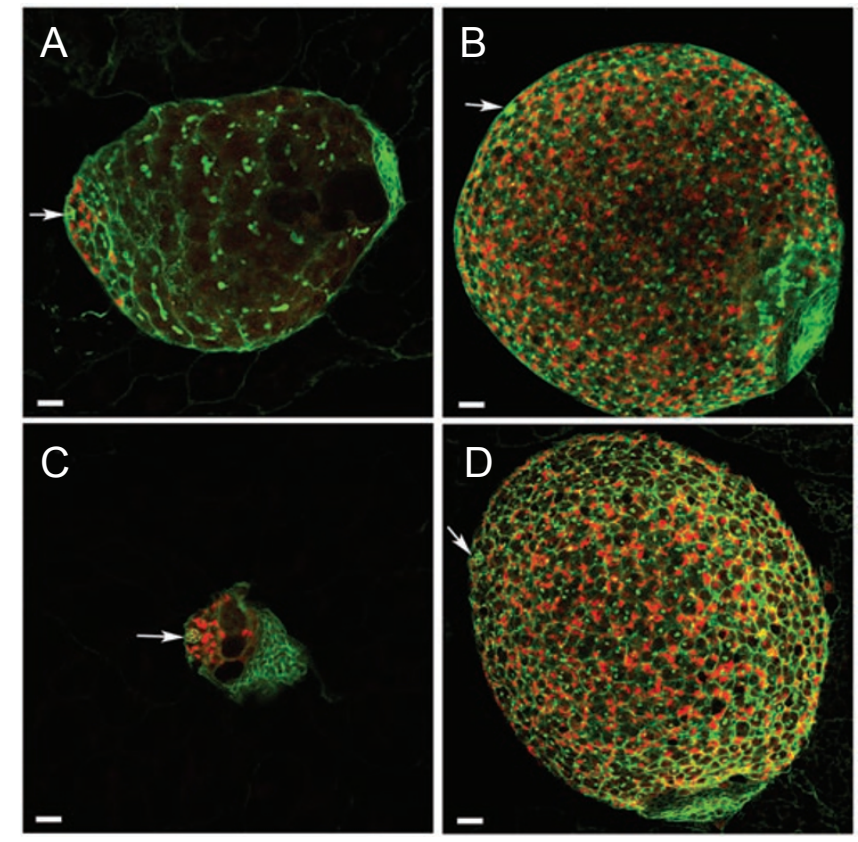
way was functional. The growth factor Upd is secreted by the hub cells and acts on GSCs to control their selfrenewal $[12,13]$. As shown in Figure 5 ectopic expression of upd in both wt and $d l g$ prevented differentiation of gonialblasts and led to the accumulation of GSCand gonialblast-like cells showing that the JAK-STAT pathway was apparently not affected in $d l g$ testes. These data indicate that $d l g$ is dispensable for the interaction between germline and somatic cells involving the JAKSTAT pathway.

To confirm that $d l g$ plays a critical role in somatic cells during their differentiation, we specifically expressed a $U A S-d l g$ transgene in either somatic or germ cells in $d l g$ testes by using specific Gal4 drivers [31]. Three drivers direct Dlg synthesis in somatic cells, including $c 833$ (Figure 6A and E), c729 (Figure 6B and F), and T155 (Figure 6C and $\mathrm{G}$ ), whereas the nanos driver directs Dlg production in germ cells (Figure 6G and $\mathrm{H}$ ). Combina- tion of $U A S-d l g$ with the somatic drivers in $d l g$ mutant restored early testis development, whereas the combination with the germline driver was ineffective (Figure 6). The $c 833$ construct drives expression of reporter genes in the hub, SSCs, and early SCCs, and c729 in SSCs, early SCCs, and testis sheath cells, whereas T155 predominantly directs expression in late SCCs [32]. All three somatic drivers in combination with $U A S$-dlg led to the formation of SCs and spermatocyte cysts containing branched fusomes in germ cells and producing Eya in somatic cells. The overall size of the rescued testes was slightly smaller than that in wt. Expression of $d l g$ driven by $c 729$ and $c 833$ produced spermatocyte cysts, which in the distal part of the testis showed a round structure instead of the scale shape characteristic of wt cysts. With T155, the larger cysts were even more distorted and their limits were poorly defined. Dlg synthesis could be detected in the rescued testes. With $c 729$ (Figure 6I-J), the
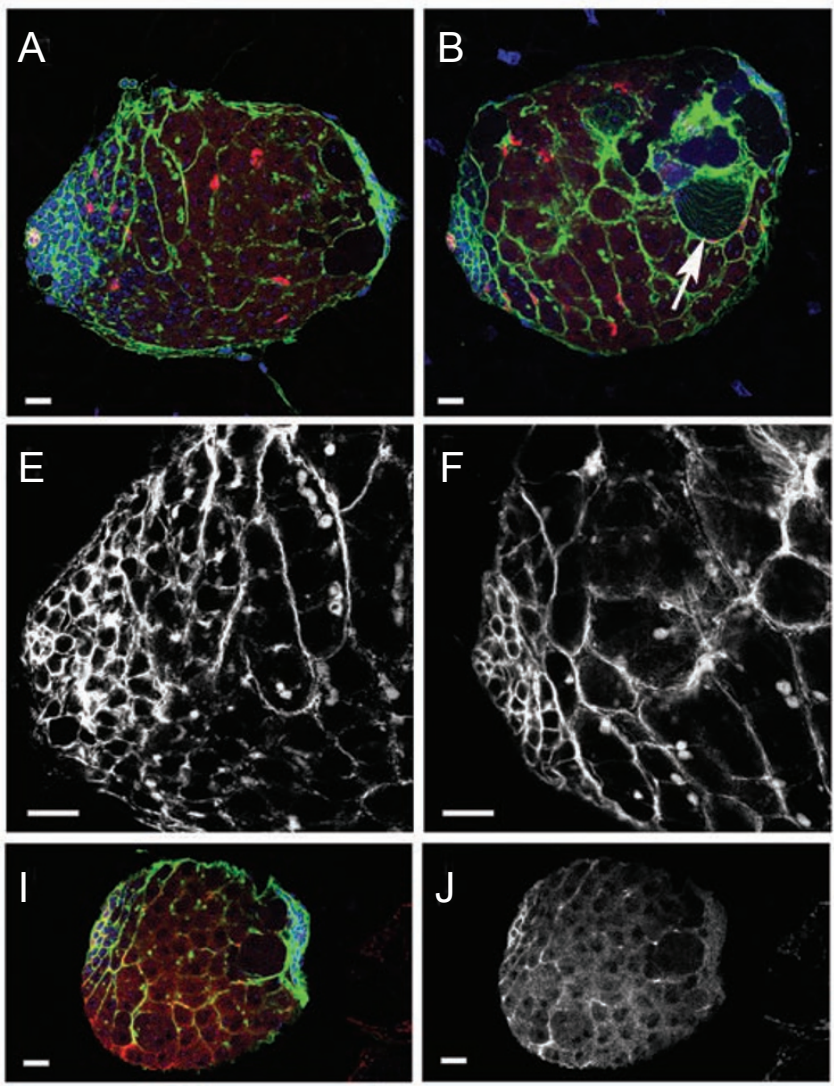
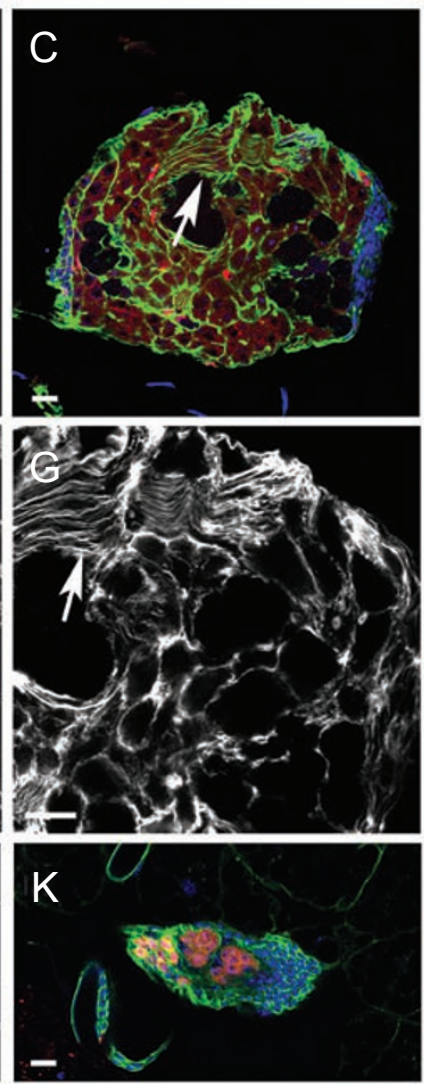
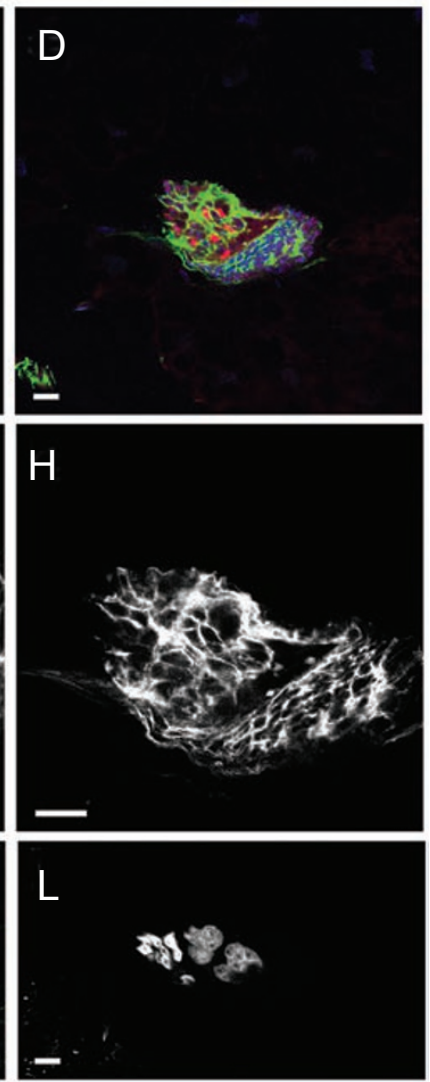

Figure 6 dlg expression in somatic cells restores testis development in mutant dlg larvae. Expression in dlg testes of UASdlg in somatic cells driven by (A, E) c833-Gal4, (B, F, I, J) c729-Gal4, (C, G) T155-Gal4, in germline cells (D, H, K, L) by nanos-Gal4. In (A-D), DNA was visualized with DAPI (blue), F-actin with phalloidin (green), the hub with Fasciclin III (red), and SCCs wrapping spermatocyte cysts with Eya (red). (E-H) Enlargements of (A-D) showing F-actin distribution. In I to L, testes were stained for Dlg (red), DNA was visualized with DAPI (blue) and F-actin with phalloidin (green). $\mathbf{J}$ and I show only the Dlg staining of $\mathbf{I}$ and $\mathbf{K}$, respectively. Testis hub is oriented towards the left. White arrows point out post-meiotic cysts with elongating spermatids. Bar: $15 \mu \mathrm{m}$. 
Dlg staining was relatively diffused between the cysts, whereas Dlg synthesis was particularly pronounced in germline cells under the nanos driver (Figure 6K-L). The c833 driver gave similar results as the c729, whereas the T155 driver gave rise to a relatively intensive staining in late SCCs (data not shown). Furthermore, in 6- to 8-dayold transgenic larvae we occasionally found cysts of elongated spermatids, indicating that meiosis had taken place (Figure 6, arrows).

\section{dlg-RNAi in SCCs blocks testis development}

To further evaluate the developmental period during which $d l g$ inactivation led to defective testis development, we silenced $d l g$ expression in somatic cells by RNA interference. For this purpose, we depleted $d l g$ transcripts by combining a UAS-dlg-RNAi transgene [33] with any of the three somatic drivers and examined the resulting phenotype. The combination of $c 833$ and $c 729$ with $U A S$-dlg-RNAi led to fully atrophied testes similar to those found in $d l g$ mutant larvae (data not shown). In contrast, combination with $T 155$ had no major effect on the initial formation of SCs, which contained branched fusomes, but affected the structure of more advanced cysts (Figure 7). In these cysts, the germ cells were predominantly individualized with fragmented fusomes forming small nodes. When $d l g$ inactivation was performed in the germ cells, no phenotype was detected (Supplementary information, Figure S1). Our data further indicated that $d l g$ function was primarily required in early SCCs, whereas $d l g$ inactivation in late SCCs led to the disruption of cyst organization.

\section{dlg overexpression triggers cyst differentiation}

In order to gain more insights into the development of cysts, we overexpressed $d l g$ in wt testes by combining the
$U A S$-dlg construct with either $c 729$ or $T 155$. Both drivers produced a similar phenotype in L3 testes (Figure 8 and Supplementary information, Figure S2). These testes were particularly filled with large cysts, and the distal somatic cells contained a marked accumulation of Dlg, which formed a scalloped fringe instead of the smooth pattern seen in wt. All together our data indicate that $d l g$ expression is particularly required in somatic cells at the beginning of cyst expansion to drive their differentiation.

\section{Discussion}

Our data demonstrate that $d l g$ acts as a key regulator of testis morphogenesis and is primarily required in cells of somatic origin. In the $d l g$ mutant testes, the SSCs and early SCCs survived as indicated by the positive $\mathrm{Tj}$ staining, whereas the late SCCs died, as revealed by the positive caspase-3 staining and the absence of Eya-staining. The critical period of $d l g$ function takes place between the time of $t j$ inactivation in somatic cells surrounding SCs [26] and that of eya activation in somatic cells wrapping spermatocyte cysts [27]. The shift between $t j$ and eya expression coincides with a marked change in Dlg distribution. Dlg is first present at the interface of all cells forming the apex of the larval testis including the hub, GSCs, SSCs, and during early stages of SC differentiation becomes restricted to the somatic cells capping the growing spermatocyte cysts.

As mutations in $t j$ encoding the Drosophila Maf transcription factor primarily prevent somatic cells to intermingle between germ cells [26], the occurrence in dlg testes of numerous Tj-positive cells mixed with germ cells indicates that $d l g$ inactivation has no effect on this process. The eya gene encodes a transcriptional activator, which in combination with Sine oculis (So), forms
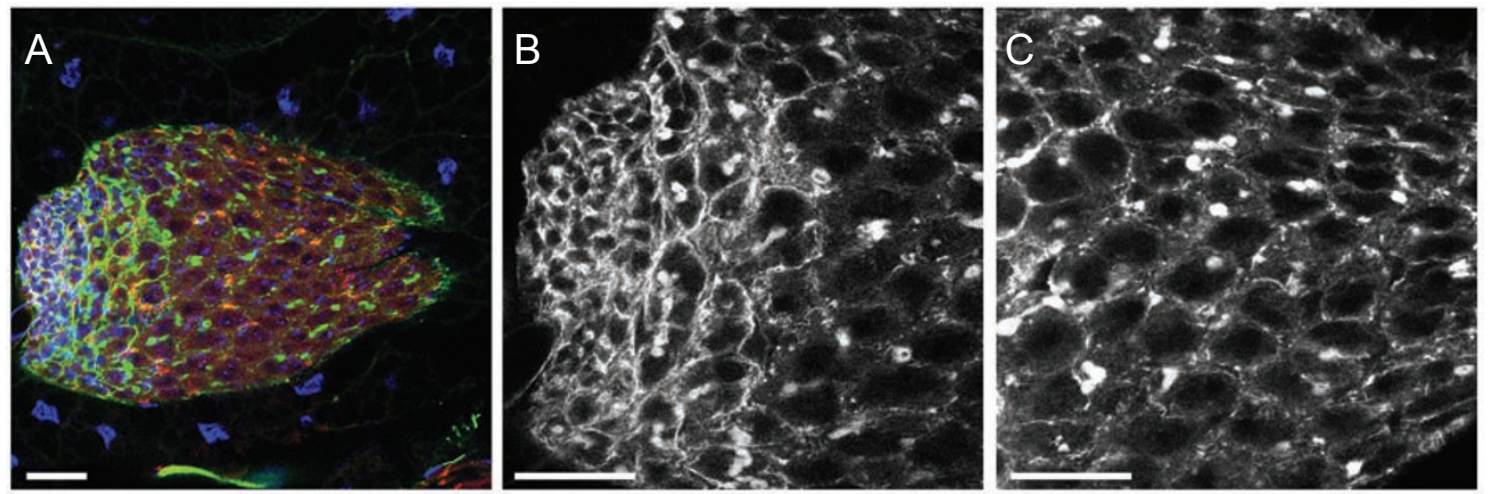

Figure 7 dlg-RNAi in SCCs blocks testis development. Depletion of dlg transcripts by UAS-dlg-RNAi driven by T155-Gal4. (A) View of a full transgenic L3 testis. The testis was stained for DNA (blue), F-actin (green), and Dlg (red). (B and C) Higher magnifications showing F-actin distribution in (B) apical and (C) distal regions of the testis shown in (A). Bar: $15 \mu \mathrm{m}$. 

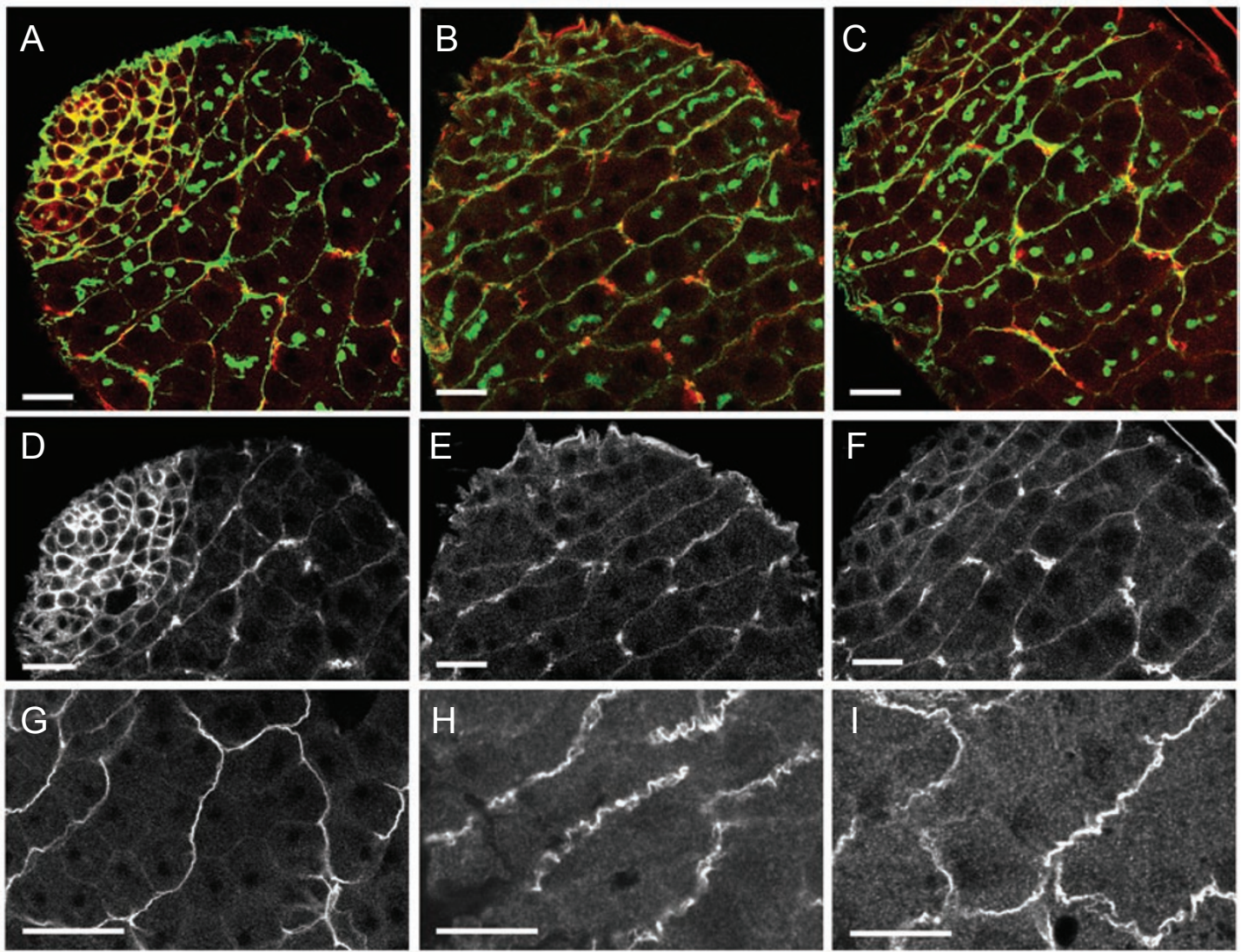

Figure $8 \mathrm{dlg}$ overexpression triggers cyst differentiation. wt testis (A) and transgenic testes expressing UAS-dlg driven by (B) c729-Gal4 and (C) T155-Gal4. Testes were stained for F-actin (green) and Dlg (red). (D-F) Dlg stained apical domains of testes shown in (A-C). (G-I) Higher magnifications of Dlg distribution at the surface of spermatocyte cysts of testes shown in (A-C). Bar: $15 \mu \mathrm{m}$.

a transcription complex required for early eye specification [34]. Clonal analysis reveals that both eya and so are required in late SCCs to ensure the survival of spermatocyte cysts during the relatively long phase of growth prior to meiosis, albeit not at the onset of spermatocyte growth [27]. Thus, eya inactivation leads to cell death in SCCs later than that produced by $d l g$. We suggest that the downregulation of eya observed in $d l g$ testes is a secondary effect of the $d l g$ mutation.

$d l g$ expression driven by $c 729$ and $c 833$ in $d l g$ mutant testes was able to restore normal development in the major part of the testes particularly around the hub and at the spermatogonial stages, whereas the pattern of spermatocyte cysts in the distal part of the testes was partially distorted. $d l g$ expression driven by $T 155$, which should predominantly occur in somatic cells surrounding spermatocyte cysts, was also able to restore development. However, the larger spermatocyte cysts were unable to aggregate in a compacted form, although some of the cysts were able to undergo meiosis. These findings dem- onstrate that germ cell development could be restored when $d l g$ was expressed in somatic cells, presumably at different stages of their development.

Depletion of $d l g$ transcripts by RNAi in SSCs and early SCCs led to the formation of fully degenerated gonads, whereas the same depletion performed in late SCCs with $T 155$ resulted in cyst fragmentation and an apparent individualization of the spermatocytes, albeit not germ cell death as observed in $d l g$ testes. The contrasted effects produced by RNAi depletion between early and late periods of cyst differentiation further show that $d l g$ plays two distinct functions during early testis development. The $T 155$ results indicate that $d l g$ acts to regulate the growth and differentiation of spermatocyte cysts. Collectively, our data support the idea that first $d l g$ acts in SSC to properly enclose growing SCs and establish functional junctions with germ cells, which are required for the survival of both germline and somatic cells. Then during a second period of testis development, $d l g$ is needed to maintain the structural integrity of the growing cysts and 
trigger polarized growth in an anterior-posterior direction. The concept of a $d l g$ requirement in cyst-polarized growth is further supported by the finding that the amount of Dlg is markedly increased in capping cells of wt cysts overexpressing $\mathrm{dlg}$. In addition, overexpression of $d l g$ by the somatic drivers led to an increased number of differentiating cysts at the testis apex, suggesting that $d l g$ acts on cyst differentiation.

Testes of $d l g$ larvae displayed a comparatively smaller number of GSCs loosely attached to the hub. The reduced rate of GSC division in $d l g$ appeared to be extrinsic and to result from the inability of SSCs to properly establish contact with GSCs, gonialblasts, and germ cells. The requirement of $d l g$ in somatic tissues is further supported by the finding that GSCs survived following RNAi depletion of $d l g$ transcripts in germ cells and, conversely, $d l g$ expression in mutant GSCs was unable to restore their viability. Although a smaller number of GSCs were detected around the hub of $d l g$ mutant testes, these cells apparently gave rise to asymmetric cell divisions generating gonialblasts, which in turn divided mitotically to form spermatogonial cells. This is in contrast to the deregulated asymmetric cell division occurring in $d l g$ neuroblasts, which divide equally due to incorrect positioning of cell polarity determinants and generate neoplasms $[6,8,35,36]$.

Known defects in asymmetric cell division of male GSCs result from an aberrant positioning of centrosomes, leading to spindle misorientation [6-8]. Mutations in genes regulating microtubule polymerization, such as centrosomin, or encoding components anchoring astral microtubules to the hub-GSC interface such as apc2, a homolog to the human tumor suppressor adenomatous polyposis coli, moderately increase the number of GSCs, which, however, maintain direct contact with the hub [5]. However, overproliferation of germ cells can operate in gonialblasts and presumably two-cell SCs. The bag-ofmarbles (bam) and benign gonial cell neoplasm (bcgn) genes act autonomously to restrict proliferation of amplifying germ cells, but not of stem cells [37]. Moreover, a recent report shows that Dlg is not among the genes that are up- or downregulated by JAK-STAT in a $b c g n$ background [38], although the Dlg protein is robustly synthesized in the hub of wt testes. These data indicate that the mechanism leading to overproliferation of male germ cells is distinct from that in neuroblasts.

$d l g$ overexpression in SCCs led to Dlg accumulation in cells capping the spermatocyte cysts. The region decorated by Dlg on the plasma membrane is clearly delineated and is spread over a limited domain of the cyst surface. The precise extension of somatic cells over germ cell cysts and Dlg distribution in these cells require more detailed studies. However, $d l g$ overexpression had no apparent influence on the spreading of somatic cells over spermatocyte cysts. We observed only a thickening of the Dlg deposit, as well as a greater waving and ruffling of the cyst cells. The cell ruffling detected in somatic cells is highly reminiscent of the formation of lammellipodialike structures upon upregulation of Rac1 in SCCs [17]. Rac1 is a downstream component of the Egfr pathway and acts antagonistically to Rho to regulate germ cell encapsulation. In the classical view of Drosophila Egfr pathway, the downstream kinase binds to the stimulated Egfr and activates a MAP-kinase cascade for transcription of target genes [39]. However, in Drosophila testis, the Egfr pathway is branched at the level of the docking protein so that binding of the adaptor protein Vav to Egfr activates the small GTPase Rac1 [17]. As the Dlg protein plays a central role in the organization of epithelial junctions and in signal transduction at sites of cellcell contact, it is possible that the C-terminal tail of Egfr interacts with one of the PDZ domains of Dlg [40, 41]. In this way $d l g$ inactivation would result in a disruption of the Egfr protein complexes at cellular junctions, block the Egfr pathway, and impair Rac1 function. Following $d l g$ overexpression, we detected an excess of differentiating cysts at the testis apex at the expense of early SCs. Egfr upregulation can result in a similar effect $[14,15]$. Based on these data, we hypothesize that Dlg may act on the cytoskeleton of the somatic cells to mediate cellshape changes leading to either cellular extensions over the SCs, or reinforcing cell-to-cell contacts with growing germ cells.

Whether our findings may be extended to higher vertebrates remains an open question. Nevertheless, in mammals like in Drosophila, spermatogenesis depends on interactions between supportive Sertoli cells and germ cells [42]. Moreover, inactivation of the Dlgh-1 gene in mice produces various abnormalities in their renal and urogenital organs with hypoplasia of kidneys and ureters, and absence of vagina and seminal vesicle [43, 44]. Generation of mice conditional for the expression of homologs of the Drosophila dlg would provide a suitable system for investigating their functions during mammalian gonadogenesis.

\section{Material and Methods}

\section{Drosophila stocks}

Oregon $R$ was used as wt. The $d l g^{m 52}$ and UAS-dlg lines were obtained from Chris Doe, UAS-upd from Georg Halder, and $d l^{X 12}$ from David Bilder. The UAS-dlg-RNAi lines were provided by the Vienna Drosophila RNAi Center (VDRC, Austria), whereas the nanos-Gal4VP16, c833-Gal4, c729-Gal4, T155-Gal4, and UASp35 lines were obtained from the Bloomington Stock Center (Indi- 
ana). Other fly stocks used in this study are described in FlyBase.

\section{Immunofluorescence staining and microscopy}

Testis immunostaining [22] was modified as follows: Testes were incubated overnight with primary antibodies in $1 \%$ PBX $(1 \%$ Triton-100X in PBS) at $4{ }^{\circ} \mathrm{C}$. Confocal images were obtained using a Leica system TCS SP2 and processed with Adobe Photoshop 7.0 .

The following antisera were used: rat polyclonal anti-Trafficjam (Tj) antibodies (1:2 000, gift from D Godt), rabbit polyclonal anti-Scrib1 antibodies [22] (1:1 000) and rabbit monoclonal caspase-3 antibody (1/1 000, Cell Signalling Technology, MA, USA). The mouse monoclonal anti-Dlg antibody was produced in hybridomal cells [45] (gift from C Doe). The mouse monoclonal antiArmadillo N7A1 (1/10), anti-FasIII (1/100), anti-eya10H6 (1/100), anti- $\alpha$-spectrin (3A9) (1/100) antibodies, and the rat monoclonal anti-Vasa (1/10) antibody were obtained from the Developmental Studies Hybridoma Bank developed under the auspices of the NICHD and maintained by The University of Iowa, Department of Biological Sciences, Iowa City, IA 52242, USA. F-actin was stained with Alexa Fluor phalloidin 488 and 546 (1/300, Invitrogen, Karlsruhe, Germany) and DNA with DAPI (Invitrogen). Following secondary antibodies were used: Alexa Fluor 647 goat anti-guinea pig $\operatorname{IgG}(1 / 300$, Invitrogen), Alexa Fluor 546 goat antirabbit $\operatorname{IgG}(1 / 300$, Invitrogen), Cy5-conjugated goat anti-mouse $\mathrm{IgG}, \mathrm{Cy} 3$-conjugated goat anti-mouse IgG and $\mathrm{Cy} 3$-conjugated goat anti-rat IgG (Jackson Immunochemistry, PA, USA).

\section{Acknowledgments}

We would like to thank the Drosophila community for generously providing us fly stocks and antibodies, in particular Chris Doe, David Bilder, Dorothea Godt, Peter Bryant, Georg Halder, the Developmental Studies Hybridoma Bank (Iowa State University), VDRC, and the Bloomington Drosophila Stock Center.

\section{References}

1 Santos AC, Lehmann R. Germ cell specification in Drosophila and beyond. Curr Biol 2004; 14:R578-R589.

2 Casper A, Van Doren M. The control of sexual identity in the Drosophila germline. Development 2006; 133:2783-2791.

3 Godt D, Tepass U. Organogenesis: keeping in touch with the germ cells. Curr Biol 2003; 13:R683-R685.

4 Fuller MT, Spradling AC. Male and female Drosophila germline stem cells: two versions of immortality. Science 2007; 316:402-404.

5 Yamashita YM, Jones DL, Fuller MT. Orientation of asymmetric stem cell division by the APC tumor suppressor and centrosome. Science 2003; 301:1547-1550.

6 Yamashita YM, Fuller MT. Asymmetric stem cell division and function of the niche in the Drosophila male germ line. Int $J$ Hematol 2005; 82:377-380.

7 Yamashita YM, Mahowald AP, Perlin JR, Fuller MT. Asymmetric inheritance of mother versus daughter centrosome in stem cell division. Science 2007; 315:518-521.

8 Gonzalez C. Spindle orientation, asymmetric division and tumour suppression in Drosophila stem cells. Nat Rev Genet 2007; 8:462-472.
9 Lin H. The stem-cell niche theory: lessons from flies. Nat Rev Genet 2002; 3:931-940.

10 Gönczy P, DiNardo S. The germ line regulates somatic cyst cell proliferation and fate during Drosophila spermatogenesis. Development 1996; 122:2437-2447.

11 Fuller MT. In: Bate M, Martinez Arias A, eds. The development of Drosophila melanogaster. Cold Spring Harbor Laboratory Press, 1993:71-147.

12 Kiger AA, Jones DL, Schulz C, Rogers MB, Fuller MT. Stem cell self-renewal specified by JAK-STAT activation in response to a support cell cue. Science 2001; 294:2542-2545.

13 Tulina N, Matunis E. Control of stem cell self-renewal in Drosophila spermatogenesis by JAK-STAT signaling. Science 2001; 294:2546-2549.

14 Kiger AA, White-Cooper H, Fuller MT. Somatic support cells restrict germline stem cell self-renewal and promote differentiation. Nature 2000; 407:750-754.

15 Tran J, Brenner TJ, DiNardo S. Somatic control over the germline stem cell lineage during Drosophila spermatogenesis. Nature 2000; 407:754-757.

16 Schulz C, Woods CG, Jones DL, Tazuke SI, Fuller MT. Signaling from germ cells mediated by rhomboid homolog stet organizes encapsulation by somatic support cells. Development 2002; 129:4523-4543.

17 Sarkar A, Parikh N, Hearn SA, Fuller MT, Tazuke SI, Schulz C. Antagonistic roles of Rac and Rho in organizing the germ cell microenvironment. Curr Biol 2007; 17:1253-1258.

18 Matunis E, Tran J, Gönczy P, Caldwell K, DiNardo S. punt and schnurri regulate a somatically derived signal that restricts proliferation of committed progenitors in the germ line. Development 1997; 124:4383-4391.

19 Woods DF, Bryant PJ. The discs-large tumor suppressor gene of Drosophila encodes a guanylate kinase homolog localized at septate junctions. Cell 1991; 66:451-464.

20 Woods DF, Hough C, Peel D, Callaini G, Bryant PJ. Dlg protein is required for junction structure, cell polarity, and proliferation control in Drosophila epithelia. J Cell Biol 1996; 134:1469-1482.

21 Bilder D, Perrimon N. Localization of apical epithelial determinants by the basolateral PDZ protein Scribble. Nature 2000; 402:676-680.

22 Li M, Marhold J, Gatos A, Török I, Mechler BM. Differential expression of two scribble isoforms during Drosophila embryogenesis, Mech Dev 2001; 108:185-190.

23 Strand D, Raska I, Mechler BM. The Drosophila lethal(2) giant larvae tumor suppressor protein is a component of the cytoskeleton. J Cell Biol 1994; 127:1345-1360.

24 Marhold J, Papagiannouli F, Li M, Patel A, Mechler BM. Requirements for scribble expression in newly formed gonads of Drosophila embryos. Gene Expr Patterns 2003; 3:143-146.

25 Lin H, Yue L, Spradling AC. The Drosophila fusome, a germline-specific organelle, contains membrane skeletal proteins and functions in cyst formation. Development 1994; 120:947956.

26 Li MA, Alls JD, Avancini RM, Koo K, Godt D. The large Maf factor Traffic Jam controls gonad morphogenesis in Drosophila. Nat Cell Biol 2003; 5:994-1000.

27 Fabrizio JJ, Boyle M, DiNardo S. A somatic role for eyes absent (eya) and sine oculis (so) in Drosophila spermatocyte 
development. Dev Biol 2003; 258:117-128.

28 Hempel LU, Oliver B. Sex-specific DoublesexM expression in subsets of Drosophila somatic gonad cells. BMC Dev Biol 2007; 7:113.

29 Hay BA, Wolff T, Rubin GM. Expression of baculovirus P35 prevents cell death in Drosophila. Development 1994; 120:2121-2129.

30 Huh JR, Guo M, Hay BA. Compensatory proliferation induced by cell death in the Drosophila wing disc requires activity of the apical cell death caspase Dronc in a nonapoptotic role. Curr Biol 2004; 14:1262-1266.

31 Brand AH, Perrimon N. Targeted gene expression as a means of altering cell fates and generating dominant phenotypes. Development 1993; 118:401-415.

32 Hrdlicka L, Gibson M, Kiger A, et al. Analysis of twenty-four lines in Drosophila melanogaster. Genesis 2002; 34:51-57.

33 Dietzl G, Chen D, Schnorrer F, et al. A genome-wide transgenic RNAi library for conditional gene inactivation in Drosophila. Nature 2007; 448:151-156.

34 Pignoni F, Hu B, Zavitz KH, Xiao J, Garrity PA, Zipursky SL. The eye-specification proteins So and Eya form a complex and regulate multiple steps in Drosophila eye development. Cell 1997; 91:881-891.

35 Albertson R, Doe CQ. Dlg, Scrib and Lgl regulate neuroblast cell size and mitotic spindle asymmetry. Nat Cell Biol 2003; 5:166-170.

36 Knoblich JA. Mechanisms of asymmetric stem cell division. Cell 2008; 132:583-597.

37 Gönczy P, Matunis E, DiNardo S. bag-of-marbles and benign gonial cell neoplasm act in the germline to restrict proliferation during Drosophila spermatogenesis. Development 1997; 124:4361-4371.

38 Terry NA, Tulina N, Matunis E, DiNardo S. Novel regulators revealed by profiling Drosophila testis stem cells within their niche. Dev Biol 2006; 294:246-257.

39 Shilo BZ. Signaling by the Drosophila epidermal growth factor receptor pathway during development. Exp Cell Res 2003; 284:140-149.

40 Humbert P, Russell S, Richardson H. Dlg, Scribble and Lgl in cell polarity, cell proliferation and cancer. Bioessays 2003; 25:542-553.

41 Funke L, Dakoji S, Bredt DS. Membrane-associated guanylate kinases regulate adhesion and plasticity at cell junctions. Ann Rev Biochem 2005; 74:219-245.

42 Fink C, Weigel R, Hembes T, et al. Altered expression of ZO-1 and ZO-2 in Sertoli cells and loss of blood-testis barrier integrity in testicular carcinoma in situ. Neoplasia 2006; 8:1019-1027.

43 Iizuka-Kogo A, Ishidao T, Akiyama T, Senda T. Abnormal development of urogenital organs in Dlgh1-deficient mice. Development 2007; 134:1799-1807.

44 Mahoney ZX, Sammut B, Xavier RJ, et al. Discs-large homolog 1 regulates smooth muscle orientation in the mouse ureter. Proc Natl Acad Sci USA 2006; 103:19872-19877.

45 Parnas D, Haghighi AP, Fetter RD, Kim SW, Goodmann CS. Regulation of postsynaptique structure and protein localization by the Rho-type guanine nucleotide factor dPix. Neuron 2001; 32:415-424.

(Supplementary information is linked to the online version of the paper on the Cell Research website.) 